in Sian, Shensi Province. Trichostrongylus orientalis has been found to be very common in Szechwan, 50 per cent of persons examined harbouring it in some localities; $11 \cdot 7$ per cent of school-children examined at Chengtu, Szechwan, were infested with Enterobius vermicularis. Ascaris lumbricoides has been found to be very widely distributed in Szechwan, with an incidence of $84 \cdot 5-95 \cdot 5$ per cent. The average worm-burden is $8-50$ worms, but some children may harbour 100-150 Ascarids. 'The fertilization of crops grown near houses with night soil is regarded as the most important epidemiological factor ; less significant are the eating of raw or partly cooked vegetables and promiscuous defecation. Under urban conditions pickled vegetables can transmit the infestation. Ascaris eggs were found to complete their embryonation after 60 days immersion in wine, vinegar, sauces, etc.

Finally, the Indian rat-flea, Xenopsylla cheopis, which is the principal vector of plague, has been found to be very common in Chengtu, Szechwan. The public health importance of flies is being studied and also the parasites of dogs, cats and other domestic animals.

It is evident that parasitologists of Free China are doing admirable work under very difficult conditions. The sympathy of everyone will go out to them, and practical help should be given from every quarter possible. There must be excellent candidates among them for the postgraduate training established at Cheloo University and the National Kiangsu Medical College.

G. LAPAGE.

\section{THE ROYAL OBSERVATORY, GREENWICH}

\section{ANNUAL REPORT}

$\mathrm{T}$ THE Annual Report of the Astronomer Royal to the Board of Visitors of the Royal Observatory, Greenwich, deals with the period May 1, 1942-April 30, 1943. Astronomical work at Greenwich has been confined to observations recently re-started on the Airy transit circle, the normal solar work on the photoheliograph and spectrohelioscope, and some special observations of the partial solar eelipse of September 10, 1942, and of certain lunar oceultations. The time service, as in the previous year, has been shared between two out-stations, the locations of which are not disclosed. Routine meteorological observations are still made at Greenwich, and the magnetic work continues at Abinger. The Chronometer Depot and the Nautical Almanac Office are also operating away from Greenwich.

Echoes of the War can be detected even in such a report as this. The Nautical Almanac Office has been engaged on computations for the Service Departments to such an extent that astronomieal work has been restricted to the minimum required for the continuance of the various almanaes. It needs little imagination to see why work has been recommenced on the "Astronomical Navigation Tables", which are being extended up to $79^{\circ} \mathrm{N}$.; while as the result of an extended visit from staff of the Director of Navigational Training (Air Ministry), modifications are to be incorporated in the "Air Almanac" which should make it even more useful to the practical navigator. New editions of the Admiralty magnetic charts have been problished, and an analysis of the residuals between observed and computed values of the various data has been undertaken to obtain information about the regions where the chart values are most uncertain. In the polar areas, where observations are few but where information is needed in war-time, the computed values are used as a guide in drawing the isomagnetic lines. Growing recognition of a close correspondence between solar activity and radio communication is emphasized by the establishment of liaison between the Observatory and certain radio organizations. The estimated times of central meridian passage of large sunspots are now telephoned, for example, to the Radio Research Station of the National Physical Laboratory, to the British Broadcasting Corporation, and to Cable and Wireless, Ltd.

It is perhaps in the section dealing with the time service that the matter of greatest general interest is to be found; here certainly the biggest changes are forecast. It is clear from this report that the Time Department of the Observatory, partly as a result of increased demands from radio communication services, partly from other war-time needs, is in process of drastic modification and extension. Experience with the dual system of operation mentioned above has clearly demonstrated the desirability of providing for two stations as a permanent measure. A Post Office line giving direct communication between the stations for 57 minutes in each hour is now available and greatly facilitates the combination of data. Short-wave signals are now radiated simultaneously with the long-wave rhythmic signals from $G B R$, and of course the 'six dots' service to the B.B.C. and the control of the Post Office 'talking elock' continue. A modification in the signal transmission procedure has been introduced for the purposes of frequency checking : the rate of the precision signals is now kept as near zero as possible. Should subsequent data show that a signal already emitted is in error (perhaps as the result of a long extrapolation), the correction will be made not gradually but in a jump, the signals then continuing with a zero rate as nearly as possible.

Two fundamental changes in the control of the time service are forecast in the report. The first concerns the standard clocks. As has been shown elsewhere (Mon. Not. Roy. Astro. Soc., 103, 196; 1943), quartz crystal clocks are affected by short-period erratics much less seriously than are pendulum clocks. The occurrenee of continual unpredictable changes of rate which, when integrated, produce disturbingly large 'wandering' in pendulum clocks can be greatly reduced by the use of quartz clocks. Prediction of elock error in cloudy weather can thus be made much more certain than it is at present, and it is already the practice to base the time service fundamentally on quartz clocks now established at other institutions, and to use the Observatory pendulum clocks as sub-standards. The report states: "The present standard of accuracy calls for the elimination of existing pendulum clocks from the operation of the time service at the earliest possible moment". The second change concerns the observational control of the standard elocks : the Astronomer Royal forecasts the supersession of visual time observations on the present standard type of transit instrument. It is believed that a photographic telescope, perhaps of the zenith tube type, should be less liable to accidental errors, and possibly also to systematic errors, than the visual instrument.

It is evident that the present trend on the time- 
keeping side of the service is towards electronic rather than electro-mechanical equipment; and on the observational side towards the impersonal photographic plate rather than the over-fallible human senses. As always, the attainment of extreme precision is costing much effort ; and if there exists any fleeting regret at the passing of the pendulum, which has served us well for nearly three centuries, it should be allayed by increasing confidence in the last decimal place of the published time-signal corrections.

\section{WAVE GUIDES IN ELECTRICAL COMMUNICATION}

$\mathrm{A}^{\mathrm{T}}$ T a meeting of the Wireless Section of the Institution of Electrical Engineers on November 3, a paper was presented by J. Kemp under the title "Wave Guides in Electrical Communication"; this having been published prior to the meeting ( $J$. Inst. Elec. Eng., 90, Part 3, No. 11 ; September 1943). With the continuous extension of radio research and application to increasingly higher frequencies, the electrical engineer is already making considerable use of wave guides in place of the more conventional pair of wires or a co-axial cable. The paper under consideration comprises a most valuable survey of the state of published knowledge on this subject and a good introduction for those who have to become familiar with this new technique in the immediate futures.

The fundamental theoretical principles of the propagation of electric waves inside hollow conductors or 'guides' have been known for nearly half a century, as a result of the investigations of Sir J. J. Thomson, Sir Joseph Larmor and particularly of Lord Rayleigh, who, in 1897, first dealt with the sukject in a comprehensive manner, and established the foundations of the theory on which the modern structure rests. Although Lord Rayleigh's investigations were restricted to hollow metal tubes of infinite conductivity, of unlimited length and of arbitrary cross-section, he was able to show that two distinct classes of waves could be propagated down such a tube. These waves are distinguished by the fact that, in addition to the transverse electric and magnetic forces which accompany the transmission of waves along two parallel wires or a co-axial cable, there is a resultant longitudinal force, which is electric in one case $(E$ class wave) and magnetic in the other case ( $H$-class wave). Corresponding to the various modes of vibration of a circular membrane or of liquid in a cylindrical vessel, each of the above two classes of wave can be propagated in wave guides in various modes and orders, the essential features of which are described and illustrated in Mr. Kemp's paper.

Up to about 1930, the further investigation of the 'guiding' action of conductors on electric waves was largely confined to the study of transmission line technique or of the propagation of waves round the surface of the earth. Owing to the fact, shown by Lord Rayleigh, that wave-guide transmission could only take place when the wave-length was of the same order or less than the cross-sectional dimensions of the tube, the practical development of the subject had to await the availability of means for the production of very short waves. The paper under consideration shows that, during the past eight years or so, much of the experimental investigation and developrnent of wave-guide technique has been carried out in the United States, notably by G. C. Southworth, S. A. Schelkunoff, W. L. Barrow and their co-workers. All this and other work are summarized in an admirable manner in Mr. Kemp's paper, which describes and illustrates the way in which the various types of waves may be launched into guides, the attenuation and other conditions to which they are subject, and the means whereby they may be converted from one type to another, or may be amplified, detected and received for practical use. In addition, by providing suitably spaced apertures in a wave guide, or by terminating it in a flared horn of suitable size and shape, the wave energy propagated along the guide may be radiated into free space in the form of a concentrated or widespread beam analogous to the emission of longer waves from antenna arrays and reflector systems. Conversely, such horns may be used to receive incoming radiation and feed it into a suitable wave-guide on its way to the radio receiver.

As the author of the paper under consideration points out, the prospective field of application of wave guides embraces various systems of communication, providing telephone and television channels in numbers vastly exceeding those of any system of established type. When used in conjunction with horns, the guides may serve for broadcasting and television, for blind landing of aircraft, for detecting, locating and manceuvring of ships, and for other purposes such as radio telephone links, for which at present radiators and receivers of conventional type are used. The full extent of the field of application of wave guides will ultimately be governed by economic as weil as technical considerations, and must therefore remain largely a matter of conjecture; but, judged from the course the development has taken during recent years, it is likely that the field will be large and attractive.

\section{ARCH/EOLOGICAL EXCAVATION IN KASHMIR}

$\mathrm{D}^{\mathrm{H}}$ R. HELLMUT DE TERRA has issued an account of his investigations of the megaliths of Bursahom, Kashmir (Proc. Amer. Phil. Soc., 85, No. 5; September 1942). The valley of Kashmir has been, of course, of the greatest importance in Indian prehistory. It has been a channel through which cultures have penetrated southwards from west-central Asia on one hand and from the Tarim basin on the other. When the early Indus civilization, about which something is now known, thanks to the excavations at Mohenjo Daro, etc., began to wane, cultures from the far north gradually penetrated into northern India, and Kashmir was one of the routes taken by the immigrants. It is to be expected, then, that any early finds in Kashmir might well show foreign influences.

Bursahom is situated on a hilltop composed of pleistocene material, and near by is a megalithic monument recalling in shape a cromlech. Excavation revealed three layers. The uppermost yielded sherds belonging to the Buddhist period of the later IndoScythian dynasty of the fourth century. The second cultural level yielded hearths, loose stone foundations and five pits with urns containing the bones of pig and deer. Black burnished ware, decorated with incised geometric ornamentation, occurs. It is suggested that this culture shows influences from north-west or west-central Asia, and that the mega- 